\title{
Contribution to the Theoretical Study of Electrical Conductivity of Materials Composites
}

\author{
Fethi Boudahri ${ }^{1}$, Abderrazzak Baba-Ahmed ${ }^{1}$, Mokhtar Bourdim ${ }^{1}$, Abdelkarim Ferouani ${ }^{2}$, \\ Walid Belkilali ${ }^{1}$ \\ ${ }^{1}$ Facuty of Science and Technology, University of Relizane, 48000 Relizane, Algeria \\ ${ }^{2}$ Higher School of Applied Sciences, Tlemcen, Algeria
}

Received: May 2, 2021. Revised: January 4, 2022. Accepted: January 22, 2022. Published: February 28, 2022.

\begin{abstract}
The present work, constituted a theoretical study of the phenomenon of the electric conductivity of composite conducting polymers. Generally, the polymers are used as electrical insulators. The incorporation of conducting loads in an insulating polymeric matrix makes it possible to obtain materials having at the same time a high electric conductivity and a low density. These materials offer a great number of applications such as the electromagnetic shielding, the protection of metals against corrosion, the adhesives conducting, the connectors, the sensors, etc. The prediction and modelling of the electrical behaviour of these composite materials are needed for the choice of their scope and could therefore reduce the onerous experimental work and the cost of production through an optimized design.

We carried out a theoretical study. To make this study, we are based on experimental results existing in the specialized literature and we build a new ideal model which describes the variation of electrical conductivity in function the voluminal fraction of the conducting loads. The comparison between our ideal model suggested and other models of McLachlan, Kirkpatrick and Landauer, shows that the model suggested is in concord with the experimental results.
\end{abstract}

Keywords-Electric conductivity, Materials composites, percolation threshold, Polymer.

\section{INTRODUCTION}

$\mathrm{P}$ olyethylene (PE) is found to be one of the most commonly used plastics in the world, belonging to the group of polyolefins [1]. Its wide applications, among others in the packaging, chemical, electrotechnical, and machine industries, result mainly from its easy processing, recyclability, and physical properties such as high wear resistance, high impact resistance, good chemical resistance, low density, physiological inactivity, and low price [1]. Additionally it does not absorb water [1]. Polyethylene (PE) is found to be one of the most commonly used plastics in the world, belonging [3]. Currently, in the cable industry, a broad range of polyolefins, alone or as blends, with various additives and conductive fillers are used as the materials to manufacture insulation and semi-conductive shields for the use in electric cables [3]. The typical construction of medium and high voltage cables includes a conductor surrounded by an insulation and semiconductive layers/screens. The inner and intermediate semiconductive layers are most often a semiconducting crosslinked polymer layer applied by extrusion around the conductive element and over the insulation layer. The insulating layer is selected from crosslinked polyethylene (XLPE), ethylene-propylene rubbers, and ethylene propylene diene rubbers (EPDM rubbers). A bonded or strippable semiconductive shield in these cables is based on low density polyethylene (LDPE), linear low density polyethylene (LLDPE) or medium density polyethylene (MDPE) compositions with ethylene-vinyl acetate, ethylene alkyl acrylate or methacrylate copolymers, and with an appropriate type and amount of carbon black (CB) [3]. In order to achieve the electrical resistivity that will meet the requirements of cable standards (below $500 \Omega \cdot \mathrm{m}$ ), such composite has to be filled with large amount of CB (up to 40 wt. \%) [3,4]. Composite materials are increasingly used in several industrial applications such as aerospace, automotive, shipbuilding and offshore oil [20]. Composite materials have high mechanical and electrical properties at low weight. Many experiencebased approaches and theoretical models have been established to predict the mechanical and electrical properties of composites materials [20]. The dielectric studies show that the synthesized samples possess high dielectric constants, high ac conductivity and low loss factors which further improve with gadolinium doping [22].

In the recent past, polymer materials have been for long time used as electrical insulators for applications requiring a lack of electrical conductivity. From the seventies, considerable interest is generated for the study of materials involving the mechanical properties of classical polymers and the electrical properties of metals to produce new materials known as conducting polymer composites. 
The electrical conductivity of polymeric materials can be increased by the addition of carbon fillers [19], such as carbon fibers, carbon black, and graphite in the polymer matrix. The resulting composites can be used in applications where metals have typically been the materials of choice. The possible applications include electromagnetic and radio-frequency interference (EMI/RFI) shielding for electronic devices and electrostatic dissipation (ESD). Electrical conductivity models are often proposed to explain and predict the conductivity behaviour of these composites [5]. Development of more accurate models would allow for more efficient materials design and could therefore reduce costly experimental work as well as reduce material and production costs through optimized design [5]. ybrid carbon nanotube composites with two different types of fillers have attracted con $\square$ siderable attention for various advantages. The incorporation of microscale secondary fillers creates an excluded volume that leads to the increase in the electrical conductivity [23].

\section{BACKGROUND}

The electrical conductivity of a composite is generally characterized by its dependence on the filler volume fraction. At low filler loadings, the conductivity of the composite is still very close to that of the pure polymer matrix. At some critical loading, called the percolation threshold, the conductivity increases several orders of magnitude with very little increase in the filler amount. After this region of drastic increase, the conductivity once again levels off and is close to that of the filler material (see fig.2). This is at the percolation threshold where enough filler has been added so that it begins to form a continuous conductive network through the composite. [6] provided a theoretical study devoted to compare the effective conductivity of the composites reinforced by graphene nanosheets (GNSs) and CNTs, which has shown that the composites reinforced by GNSs have a lower percolation threshold and higher conductivity and critical exponent, and can form a conductive network more easily than those reinforced by CNTs with the same volume fraction. However, our observations are more similar to those made by $\mathrm{Du}$ et al. [7], who obtained for HDPE/GNSs nanocomposites the percolation threshold of 1 vol. Various models have been proposed in an effort to predict the electrical conductivity behavior of composites based on numerous factors. While all the models base calculations on the filler volume fraction, there are other factors that can affect the conductivity of the composite, as well as the volume fraction at which the percolation threshold occurs. Physical properties of both the filler and the polymer will influence the composite and include structural properties, interfacial properties of two constituents $[5,8]$.

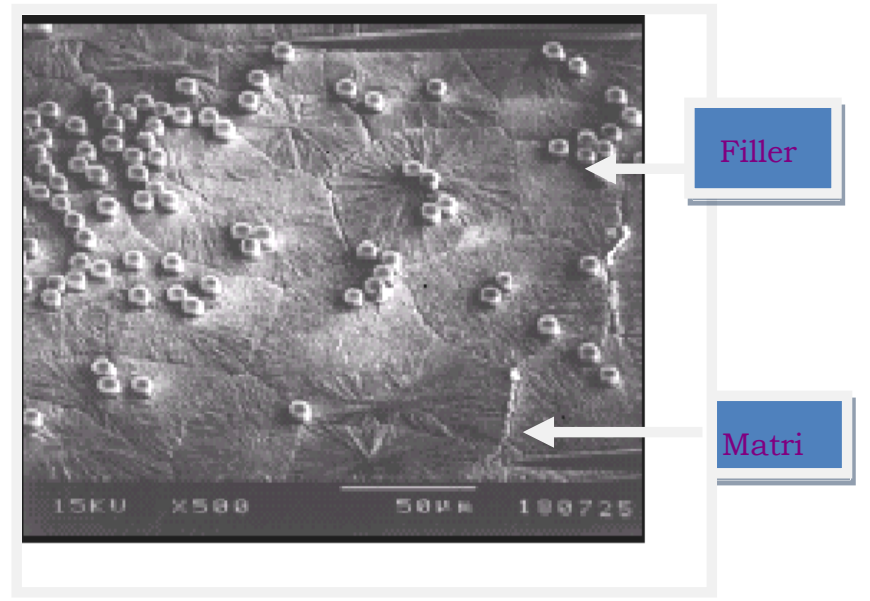

Figure 1. Analysis microstructure of a composite material

Typical conductivity values for polymers range from 10-14 to $10-17$ Siemens $/ \mathrm{cm}(\mathrm{S} / \mathrm{cm})$. In contrast, the electrical conductivity of carbon fillers can range from 102 to $104 \mathrm{~S} / \mathrm{cm}$, compared to metals, which are typically around $106 \mathrm{~S} / \mathrm{cm}$. The different forms of carbon, such as fibers or carbon black, usually have different inherent conductivity, and it is this value that typically controls the upper bound of the conductivity curve. In the region of a higher filler amount, the composite's conductivity should level off to a value equal to or slightly lower than that of the filler. Ultimately, the value at which the conductivity levels off at is often dependent on other properties of the composite as well [9].

This is at the percolation threshold where enough filler has been added so that it begins to form a continuous conductive network through the composite.

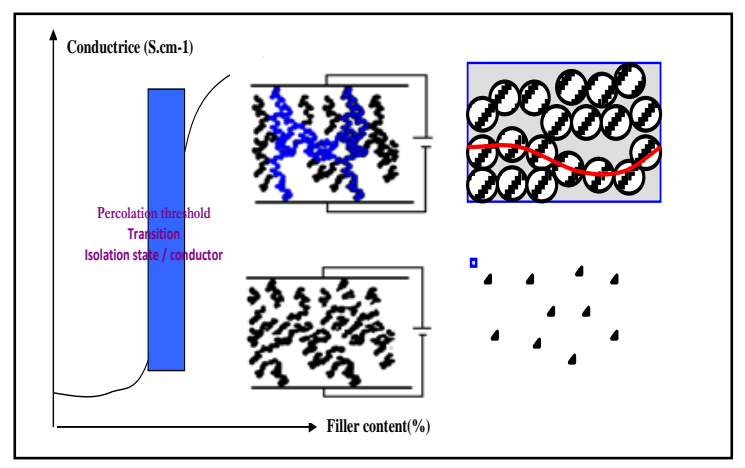

Figure 2. Diagram of the transition insulator / conductor in conductive polymers.

Zone A: domain of insulation.

Zone B: domain of percolation.

Zone C: domain of high conduction

Understanding the mechanisms responsible for electrical conduction in conductive polymer composites is essential both for controlling this type of material that needs to meet the technical and industrial. Today, there are several theoretical 
models, like those of Mclachlan, and Kirkpatrick, which allow us to explain certain properties of the electrical transport in conducting polymer composites, but by definition a model is still incomplete and imperfect.

To this end, we propose a new model for improving the evaluation of the electrical conductivity of conductive polymer composites based on the volume fraction of conductive inclusions.

\section{THEORETICAL MODELS}

To date, several theoretical models have been proposed to explain and predict the variation of electrical conductivity in conductive polymer composites.

\section{A. Kirkpatrick Model}

The model proposed by Kirkpatrick predicts the electrical conductivity from the contact of the particles within the composite.

a network of points, of the face-centred cubic type, is used to determine the percolation concentration. by simulation, it is possible to calculate the points and the links which are in a cluster and when the latter extends to the limits of the systems. Thus it is possible to predict the percolation threshold by converting the predicted values into a volume fraction

The model that was proposed followed a power-law equation of the following form [13], [21]:

$$
\sigma=\sigma_{0}\left(\phi-\phi_{C}\right)^{S}
$$

where $\sigma$ is the conductivity of the mixture; $\sigma 0$, the conductivity of the filler $\Phi$, the volume fraction of the filler; $\Phi$ c the volume percolation fraction; and the critical exponent $\mathrm{s}$, dependent upon the dimension of the lattice.

This particular model was not completely accurate in calculating the electrical conductivity. However, it has become the basis for many of the later conductivity models. As a result, they have become slightly more accurate predictors of electrical conductivity [13].

\section{B. McLachlan Model}

McLachlan suggested that this particular model could be used for any system comprising a conducting material, an example of which is the system studied here. This model is based on a general effective media equation and is similar in form to a statistical model. The equation of interest is similar in form to a statistical model, which takes into account the conductivities of constituent materials and is given by [15], [16],[18]:

$$
\frac{\left(1-\varphi_{r}\right)\left(\sigma_{p}^{1 / t}-\sigma^{1 / t}\right)}{\sigma_{p}^{1 / t}+\left[\frac{\left(1-\varphi_{c}\right)}{\varphi_{c}}\right] \sigma^{1 / t}}+\frac{\varphi_{r}\left(\sigma_{r}^{1 / t}-\sigma^{1 / t}\right)}{\sigma_{r}^{1 / t}+\left[\frac{\left(1-\varphi_{c}\right)}{\varphi_{r}}\right] \sigma^{1 / t}}=0
$$

where $\sigma, \sigma p$ and $\sigma \mathrm{r}$ are the electrical conductivities of the polymer composite; the matrix polymer and the filler, respectively, $\Phi$ is the volume fraction of filler; $\Phi c$, the percolation threshold; and t, the critical exponent. In this model, the critical exponent, $t$, can be determined either by a calculation or by curve fitting techniques.

\section{Landauer Model}

The model proposed by Landauer predicts the electrical conductivity from the equation [13]:

$$
\sigma=\frac{1}{2}(3 \phi-1) \sigma_{r}
$$

$\sigma$ : Electrical conductivity of polymer composite conductor.

$\sigma_{\mathrm{r}}$ : Electrical conductivity of conducting inclusions(filer).

: The volume fraction of filler.

\section{Marko leveij Model}

In most cases, the relationship between measured values and measurement variables is nonlinear. Nonlinear curve fitting also seeks to find those parameter values that minimize the deviations between the observed y values and the expected $y$ values. In nonlinear models, however, one usually cannot solve the equation for the parameters [14]. Various iterative procedures are used instead. Nonlinear fitting always starts with an initial guess of parameter values. One usually looks at the general shape of the model curve and compares it with the shape of the data points [14]. For example, look at the sigmoïdal function in Fig. 2 which is identical in all gaits which describe the variation of electrical conductivity of a polymer composite conductor according to the volume fraction of filler [14].

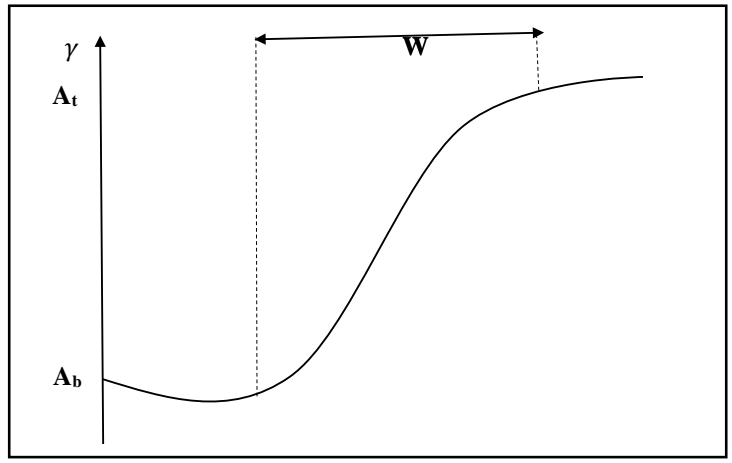

Figure 3. In nonlinear curve-fitting, such as this sigmoidal function, to minimize the deviations between the observed and expected y values, it is necessary to estimate parameter values and use iterative procedures.

$$
\gamma=\mathrm{A}_{\mathrm{b}}+\frac{\mathrm{A}_{\mathrm{t}}-\mathrm{A}_{\mathrm{b}}}{1+\exp \left(-\left(\frac{\mathrm{X}-\mathrm{X}_{0}}{\mathrm{~W}}\right)\right)}
$$




\section{RESULTS AND DISCUSSION}

For this study, we based on experimental results reported in the literature on conducting polymer composites.

In a first step, we review two composite materials with two different matrices, one high-density polyethylene (HDPE) and one low density polyethylene (LDPE), reinforced by the same charges conductive vanadium trioxide $\left(\mathrm{V}_{2} \mathrm{O}_{3}\right)$ (Tables 1 and 2) [11].

In a second step, we are interested in the variation of electrical conductivity of two composite materials consisting of a polymer matrix LDPE reinforced by successively EC (Printex XE2 Extra-conductive black, product par Degussa AG (Frankfurt, Germany)) and S6 (Sakap6 carbon filler, fabriqué par Carbochem (Gliwice, Poland)) (Tables 3-4) [12] and and we have other experimental results (Tables 5-6) [15], [17].

\section{A. CASE HDPE $/ \mathrm{V}_{2} \mathrm{O}_{3}$}

Table 1. Values of Electrical Conductivity and Volume Fraction of Filler in the Case of Composite (HDPE / $\mathrm{V}_{2} \mathrm{O}_{3}$ ) by Xiao-su Yi et al [11].

\begin{tabular}{|c|c|}
\hline $\boldsymbol{\Phi}$ & $\log \boldsymbol{\sigma}\left(\mathrm{S.cm}^{-1}\right)$ \\
\hline 0.00092 & -10.571 \\
\hline 0.08863 & -9.73779 \\
\hline 0.19774 & -9.54127 \\
\hline 0.25742 & -7.01011 \\
\hline 0.28608 & -3.04043 \\
\hline 0.30568 & -2.21505 \\
\hline 0.34400 & -0.82369 \\
\hline 0.41479 & -0.05334 \\
\hline
\end{tabular}

\section{B. CASE LDPE/V2O3}

Table 2. Values of Electrical Conductivity and Volume Fraction of Filler in the Case of Composite (LDPE / $\mathrm{V}_{2} \mathrm{O}_{3}$ ) by Xiao-Su Yi Et Al [11].

\begin{tabular}{|c|c|}
\hline $\boldsymbol{\Phi}$ & $\log \boldsymbol{\sigma}\left(\mathbf{S . c m}^{-1}\right)$ \\
\hline 0.00092 & -12.41830 \\
\hline 0.08863 & -11.99522 \\
\hline 0.19804 & -11.96019 \\
\hline 0.23606 & -11.84795 \\
\hline 0.29398 & -10.72039 \\
\hline 0.36477 & -4.99775 \\
\hline 0.39373 & -2.41943 \\
\hline 0.42415 & -1.24031 \\
\hline 0.49348 & -0.61931 \\
\hline
\end{tabular}

\section{CASE LDPE/ Sakap6}

Table 3. Values of Electrical Conductivity and Volume Fraction of Filler in the Case of Composite (LDPE / Sakap6) by Z. Garncarek \& $\mathrm{Al}[12]$.

\begin{tabular}{|c|c|}
\hline $\boldsymbol{\Phi}$ & $\log \boldsymbol{\sigma}\left(\mathbf{S . c m}^{-1}\right)$ \\
\hline 0.00066 & -14.40482 \\
\hline 0.14141 & -13.65244 \\
\hline 0.1836 & -5.32551 \\
\hline 0.19992 & -2.04090 \\
\hline 0.22700 & -1.53565 \\
\hline 0.25207 & -1.24368 \\
\hline
\end{tabular}

\section{CASE LDPE/EC}

Table 4. Values of Electrical Conductivity and Volume Fraction of Filler in the Case of Composite (LDPE / EC) by Z. Garncarek \& Al [12].

\begin{tabular}{|l|l|}
\hline $\boldsymbol{\Phi}$ & $\log \boldsymbol{\sigma}\left(\mathrm{S.cm}^{-1}\right)$ \\
\hline 0.02854 & -14.05458 \\
\hline 0.04680 & -5.21898 \\
\hline 0.12319 & -0.54745 \\
\hline 0.17284 & -0.14318 \\
\hline
\end{tabular}

\section{E. CASE NYLON6/Graphite}

Table 5. Values of Electrical Conductivity and Volume Fraction of Filler in the Case of Composite (NYLON6/ GRAPHITE) by W. Wengui \& $\mathrm{Al}[15]$.

\begin{tabular}{|c|c|}
\hline $\boldsymbol{\Phi}$ & $\log \boldsymbol{\sigma}\left(\mathbf{S . c m}^{-1}\right)$ \\
\hline 0.0007 & -14.86012 \\
\hline 0.005 & -13.82973 \\
\hline 0.007 & -7.05453 \\
\hline 0.01 & -4.94692 \\
\hline 0.026 & -3.08460 \\
\hline 0.031 & -2.88000 \\
\hline
\end{tabular}

\section{F. CASENylon6/Zn}

Table 6. Values of Electrical Conductivity and Volume Fraction of Filler in the Case of Composite (NYLON6 /ZN) by G. Pinto \& Al [17].

\begin{tabular}{|c|c|}
\hline $\boldsymbol{\Phi}$ & $\log \boldsymbol{\sigma}\left(\mathbf{S . c m}^{-\mathbf{1}}\right)$ \\
\hline 0.001 & -12.91100 \\
\hline 0.018 & -12.65400 \\
\hline 0.064 & -12.71800 \\
\hline
\end{tabular}




\begin{tabular}{|l|l|}
\hline 0.089 & -12.65400 \\
\hline 0.119 & -12.61800 \\
\hline 0.158 & -12.53900 \\
\hline 0.208 & -10.19100 \\
\hline 0.238 & -6.71200 \\
\hline 0.273 & -3.77700 \\
\hline 0.363 & -2.05100 \\
\hline 0.460 & -1.98700 \\
\hline
\end{tabular}

Our study is an extension of the Model Marko Leveij given by the following equation:

$$
\gamma=\mathrm{A}_{\mathrm{b}}+\frac{\mathrm{A}_{\mathrm{t}}-\mathrm{A}_{\mathrm{b}}}{1+\exp \left(-\left(\frac{\mathrm{X}-\mathrm{X}_{0}}{\mathrm{~W}}\right)\right)}
$$

This function has four parameters. $A_{b}$ and $A_{t}$ are two asymptotic values - values that the function approaches but never quite reaches - at small and large $X$. The curve crosses over between these two asymptotic values in a region of $X$ values whose approximate width is $\mathrm{W}$ and which is centered around $X_{0}$. For the initial estimate of the values of $A_{b}$ and $A_{t}$, we can use the average of the lower third and the upper third of the $\boldsymbol{\gamma}$ values of data points. The initial value of $\mathrm{X}_{0}$ is the average of $X$ values of the crossover area, and the initial value of $\mathrm{W}$ is the width of the region of points between the two asymptotic values.

And after a thorough study of experimental results [11], [12] describing the variation of electrical conductivity of conductive polymer composites based on the volume fraction of filler, on propose the following model:

$$
\log \sigma=\log \sigma_{r}+\frac{\left(\log \sigma_{p}-\log \sigma_{r}\right)}{\left(1+\exp \left(10 \frac{\varphi-\varphi_{c}}{\varphi_{r}}\right)\right)}
$$

With

$\sigma$ : Electrical conductivity of polymer composite conductor. $\sigma_{\mathrm{r}}$ : Electrical conductivity of conducting inclusions(filer). $\sigma_{\mathrm{p}}$ : Electrical conductivity of matrix polymer.

$\Phi$ : The volume fraction of filler. $\Phi_{c}$ : The volume percolation fraction.

\section{COMPARISON OF MODELS}

The comparison of results of the proposed model to experimental results [11-12] and their comparison with those of Kirkpatrick, Landauer and Mclachlan is needed to validate our model.

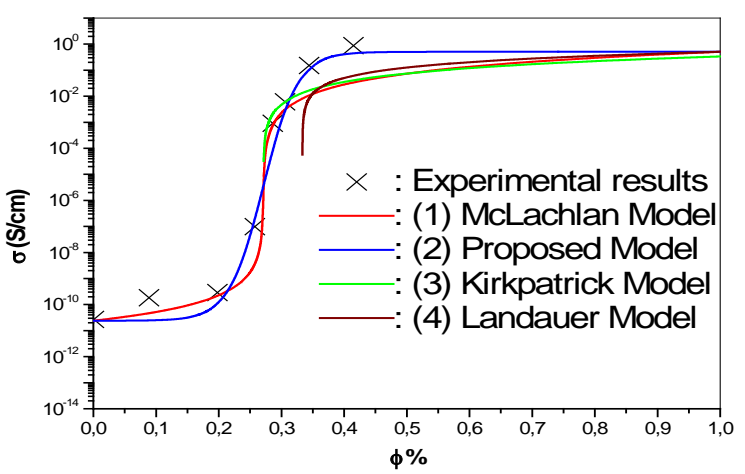

Figure 4. Electrical conductivities of the HDPE/V2O3 composite as a function $\mathrm{V}_{2} \mathrm{O}_{3}$ content

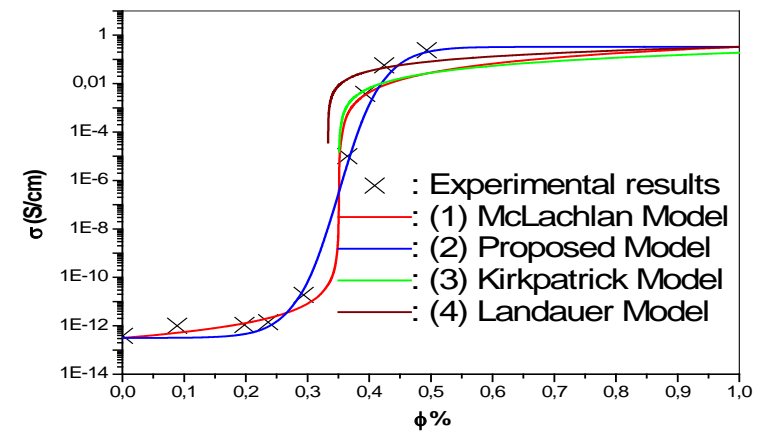

Figure 5. Electrical conductivities of the LDPE/V2O3 composite as a function $\mathrm{V}_{2} \mathrm{O}_{3}$ content.

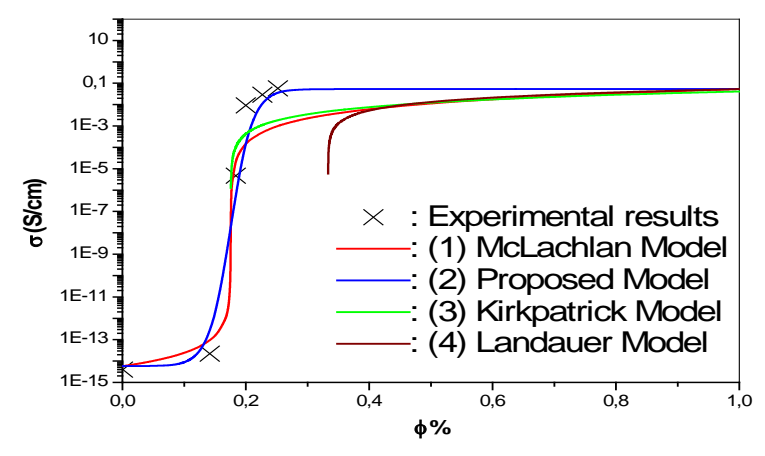

Figure 6. Electrical conductivities of the LDPE/ $\mathrm{S}_{6}$ composite as a function $\mathrm{S}_{6}$ (Sakap6 carbon filler ) content

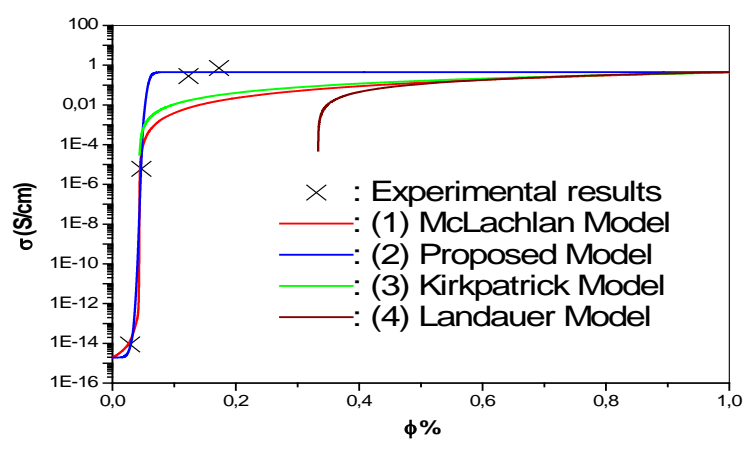

Figure 7. Electrical conductivities of the LDPE/EC composite as a function EC content. 
Fig (4-7), represents the variation of electrical conductivity depending on the filler volume fraction for four composite materials considered.

These curves are given by the model McLachlan, Kirkpatrick, Landauer end proposed model.

The results given by the proposed model check three areas predicted by the theory of percolation. Examination of the curves obtained shows that those resulting from the proposed model agree well with experimental results.

The model also checks Mclachlan three area mentioned above, but underestimates the value of the electrical conductivity of the composite in the saturation zone $(\Phi>\Phi \mathrm{s})$.

The Kirkpatrick model is limited to the study of variation of electrical conductivity of composites in the $\Phi>\Phi c$

The difference given by the Landauer model compared to experimental results is important. This difference is due to the fact that this model sets a prior critical volume fraction equal to $1 / 3$ for all conductive polymer composites studied. Moreover, it was only predicted that the electrical conductivities for the filler volume fraction above 1/3. Like the other two models, the Landauer underestimates the values of electrical conductivity in the $(\Phi>\Phi s)$.

\section{CONCLUSION}

This work is a contribution to the theoretical study of the electrical conductivity of conducting polymer composites. Modeling the variation of electrical conductivity of conducting polymer composites is necessary for the choice of their scope and could facilitate the implementation of these materials and to optimize cost. Modeling the variation of electrical conductivity of in this work, we contribute to the development of theoretical models to describe the change in electrical conductivity of conductive polymer composites based on the volume fraction of conductive inclusions.

We propose for it a new theoretical model describing the change in electrical conductivity of conductive polymer composites based on the volume fraction of reinforcement. To validate the proposed model, we conducted a comparative study of results obtained by the proposed model with those predicted by the model Mclachlan, Kirkpatrick and Landauer. The results of the proposed model are in good agreement with experimental results.

\section{ACKNOWLEDGMENT}

I would like to express my special thanks of gratitude to my professor Kara Kamel as well as our principal Mohammed Bennekrouf who gave me the golden opportunity to do this wonderful project.

Secondly i would also like to thank my parents and friends who helped me a lot in finalizing this project.

\section{References}

[1] Sandra Paszkiewicz, Anna Szymczyk, Daria Pawlikowska, Jan Subocz, Marek Zenker, and
Roman Masztak. "Electrically and hermally conductive Low Density Polyethylene-Based Nanocomposites Reinforced by MWCNT or Hybrid MWCNT/Graphene Nanoplatelets with Improved Thermo-Oxidative Stability." Nanomaterials (Basel), v.8(4), 2018 Apr; 264..

[2] Kulshreshtha, A.K.; Talapatra, S. Competitive New Technologies in Polyolefin Synthesis and Materials. In Handbook of Polyolefins, 2nd ed.; $\quad$ Vasile, C., Ed.; CRR Press: Boca Raton, FL, USA, 2000.5.

[3] Cousins K. Polymers for Wire and Cable Changes within an Industry. Smithers Rapra chnology; Shawbury, UK: 2000. A Rapra Industry Analysis Report.

[4] Belli S., Bareggi A., Dell’Anna G., Scelza C., Donazzi F. Continuous process for manufacturing electrical cables. 205143. PAT. 2003 Mar 31.

[5] Matthew L. Clingerman, Julia A. King, Kirk H. Schulz, Jeffrey D. Meyers, Evaluation of electrical conductivity models for conductive polymer composites, Journal of applied polymer science, vol. 83, 1341-1356 (2003).

[6] Xie, S.H.; Liu, Y.Y.; Li, J.Y. Comparison of the effective conductivity between composites reinforced by graphene nanosheets and carbon nanotubes. Appl. Phys. Lett. 2008, 92, 24312.

[7] Du, J.; Zhao, L.; Zeng, Y.; Zhang, L.; Li, F.; Liu, P.; Liu, C. Comparison of electrical properties between multiwalled carbon nanotube and graphene nanosheet/high density polyethylene composites with a segregated network structure. Carbon 2011, 49, 1094-1100.

[8] Nicoleta-Violeta Stanciu, Felicia Stan , Thermal, Rheological,Mechanical, and Electrical Properties of Polypropylene/Multi-Walled Carbon Nanotube Nanocomposites. Polymers 2021, 13, 187.

[9] Celzard, A.; Marêché, J.F.; Furdin, G.; Puricelli, S. Electrical conductivity of anisotropic expanded graphitebased monoliths. J. Phys. D Appl. Phys. 2000, 33, 30943101.

[10] J. C. Dubois, Polymères conducteurs, Techniques de l'ingénieur, traité Electronique E 1 860, Université Pierre et Marie Curie (1999).

[11] Xiao, S. Yi, G. Wu and Y. Pan - "Properties and applications of filled conductive polymer composites"Polymer International Vol. $44 \mathrm{~N}^{\circ} 2$ (1997).

[12]Z. Garncarek, R. Piasecki, J. Borecki, A. Maj and M. Sudol, "Effective conductivity in association with model structure andspatial inhomogeneity of polymer/carbon black composites”, Appl. Phys.29 1360 - 1366 (14 may 1996).

[13] Y. Zweifel, Approche expérimentale des phénomènes microscopiques de la conduction électrique dans les polymères chargés, thèse de doctorat, Lausanne EPLF (1996).

[14] Marko Ledvij, 24 Industrial Physicists, American Institute of Physics, April/may 2003.

[15] W. Wengui, C. Guohua, W. Dajun, Transport properties of electrically conducting nylon6/foliated graphite nanocomposites, polymer, 46 (2005) 6250-6257.

[16]D. S McLachlan, Kfeng Cai, G. Sauti, AC and DC conductivity- based microstructural characterization, 
International journal of Refectory Metal and Hand Materials, 19 (2001) 437-445.

[17] G. Pinto and A.J Martjn, Conducting Aluminieum- filled Nylon6 composites, Polymer composite, February 2001, vol.22,1.

[18]K. kara zaitri, S khaldi, M. hamouni, f. Boudahri; Application du modèle de Mclachlan pour l'étude de la conductivité électrique des composites polymères conducteurs; Workshop international sur la condensation de Bose-Einstein et la physique des polymères; chelef ; february 2007.

[19] F. Boudahri and al; effet de la nature du renford sur la conductivité électrique des polymers conducteurs composites; International conference on modeling and simulation ; july 2007.

[20] Soufiane Belhouideg, Prediction of effective mechanical properties of composite materials using homogenization approach: Application to tungsten fiber reinforced bulk metallic glass matrix composite ;International Journal of Materials; Volume 5, 2018.

[21]S. Yamashitaa , i. Ohsawaa and j. Takahashi ;Experimental and Theoretical Study about Electrical and Thermal properties of carbon fiber mat reinforced thermoplastics; eccm16 - 16 th european conference on composite materials, seville, spain, 22-26 june 2014.

[22] Ramanjeet Kaur, Anand K Tyagi; Structural, Morphological and Dielectric Studies of Sol Gel Derived Gd Doped SrTiO3- $\delta$; International Journal of Materials ;Volume 7, 2020.

[23] Sung-Hoon Park, Jinyoung Hwang, Gyeong-Su Park, JiHwan Ha , Minsu Zhang, Dongearn Kim, Dong-Jin Yun, Sangeui Lee \& Sang Hyun Lee ; Modeling the electrical resistivity of polymer composites with segregated structures; Nature Communications | (2019) 10:2537;
Contribution of Individual Authors to the Creation of a Scientific Article (Ghostwriting Policy)

F. Boudahri performed the modeling with Maple and origine.

A. Baba Ahmed has organized and theoretical study of the different models.

M. Bourdim and W. Belkilali organized and discussed the results.

Creative Commons Attribution License 4.0 (Attribution 4.0 International, CC BY 4.0)

This article is published under the terms of the Creative Commons Attribution License 4.0

https://creativecommons.org/licenses/by/4.0/deed.en_US 